Bull. Austral. Math. Soc.

$16 \mathrm{~S} 40,57 \mathrm{~T} 05$

VOL. 72 (2005) [109-127]

\title{
FINITE PRESENTABILITY OF SOME METABELIAN HOPF ALGEBRAS
}

\author{
Dessislava H. Kochloukova
}

\begin{abstract}
We classify the Hopf algebras $U(L) \# k Q$ of homological type $F P_{2}$ where $L$ is a Lie algebra and $Q$ an Abelian group such that $L$ has an Abelian ideal $A$ invariant under the $Q$-action via conjugation and $U(L / A) \# k Q$ is commutative. This generalises the classification of finitely presented metabelian Lie algebras given by J. Groves and $\mathrm{R}$. Bryant.
\end{abstract}

\section{INTRODUCTION}

The purpose of this paper is to try to unite some existing methods used in the classification results of metabelian Lie algebras and metabelian discrete groups of homological type $F P_{2}$ via the language of Hopf algebras. This sheds more light on the similarities between the Lie and group cases and explains partially the differences. Still some of the results in the group case have homotopical flavour, using methods from covering spaces to establish that having homological type $F P_{2}$ imposes strong condition on the first $\Sigma$ invariant of the group ([4]). These methods do not have a purely algebraic counterpart. The Lie case was treated in $[5,6]$ with algebraic methods, and a Lie invariant (with a valuation flavour) for metabelian Lie algebras was proposed. This plays the same role in the Lie theory as the Bieri-Strebel $\Sigma$-invariant for metabelian groups. In this paper we do not suggest a new invariant but establish that the main result of [5] holds for some metabelian Hopf algebras. It is interesting to note that in both the Lie and group cases calculations with the second homology group of Abelian objects (Lie algebras or Abelian groups) viewed as modules over a commutative ring via the corresponding diagonal action was always quite helpful. The definition of the diagonal Lie and group actions can be united via the comultiplication map of Hopf algebras, and this was the starting point of our considerations.

We study Hopf algebras $H=U(L) \# k G$ over a field $k$, that is, smash products of universal enveloping algebras $U(L)$ of Lie algebras $L$ over $k$ by group rings $k G$, where $G$ acts via conjugation on $L$ and write $\mathcal{X}$ for the category of such Hopf algebras. This category is quite important. If $\operatorname{char}(k)=0$ it coincides with the category of cocommutative,

Received 21st March, 2005

Partially supported by "bolsa de produtividade de pesquisa" from CNPq, Brazil.

Copyright Clearance Centre, Inc. Serial-fee code: 0004-9727/05 \$A2.00+0.00. 
pointed Hopf algebras over $k[8,5.6 .4,5.6 .5]$. Still in this paper we do not impose any condition on the characteristic of $k$.

By the main results of $[\mathbf{5}, \mathbf{6}]$ for metabelian Lie algebras the homological property $F P_{2}$ is equivalent to finite presentability (in the Lie sense). Finite presentability in the category $\mathcal{X}$ was defined in [7] and is further explained in Section 1 . The following theorem classifies some metabelian Hopf algebras in $\mathcal{X}$ and shows that again finite presentability and the property $F P_{2}$ coincide. It is well known that the properties $F P_{2}$ and finite presentability are not equivalent for general groups ([2]), but the same problem is open for Lie algebras.

THEOREM 1. Let $H=U(L) \# k Q$ be a finitely generated Hopf algebra with $A$ an $Q$-invariant (via conjugation) Abelian ideal in the Lie algebra $L$ such that $R=U(L / A) \# k Q$ is commutative. Then the following are equivalent:

1. $\quad H$ is finitely presented in the category $\mathcal{X}$;

2. $H$ as associative ring is of homological type $F P_{2}$;

3. $A \wedge A$ is finitely generated right $R$-module, where $R$ acts on $A$ via the adjoint action and $R$ acts on $A \otimes A$ via the comultiplication $\Delta: R \rightarrow R \otimes R$;

4. $A \otimes A$ is finitely generated right $R$-module, where $R$ acts on $A \otimes A$ via the comultiplication $\Delta$.

The proof of the above theorem will be given by showing that every condition implies the following and that 4 implies 1 . The most difficult part of the proof is 4 . implies 1 . and is done in Theorem 4. The proof of Theorem 4 is quite long. It partially follows the method introduced in [5]. Still our set of relations that will show that $H$ is finitely presented is much larger than the one considered in [5] (even if we are in the Lie case $Q=1$ ), but by considering more relations we manage to simplify the argument from [5]. In fact a blind translation of the method of [5] in the Hopf case does not work because the comultiplication $\Delta$ sends group-like elements $g$ to $g \otimes g$, thus increases the length of elements and a big part of the proofs in [5] is based on induction on length.

Finally we observe that Theorem 1 is an extension of the main results of [5] and [6] but the main result about metabelian groups of [4] does not follow from Theorem 1. It will be interesting to find a description of the Hopf algebras $H=U(L) \# k G$ of type $F P_{2}$, where $H$ is a Hopf extension in the category $\mathcal{X}$ of two commutative smash products (in particular this implies that $G$ is metabelian) and find a new invariant that generalises simultaneously the Bieri-Strebel invariant [4] and the Bieri-Groves invariant [6]. 
1. Preliminaries on the category $\mathcal{X}$ and finite presentability in $\mathcal{X}$

The category $\mathcal{X}$ is a subcategory of the category of Hopf algebras over $k$. An object of $\mathcal{X}$ is a smash product

$$
\begin{gathered}
H=U(L) \# k G \simeq U(L) \otimes k G, \\
L=\{a \in H \mid \Delta(a)=a \otimes 1+1 \otimes a\}, G=\{g \in H \mid \Delta(g)=g \otimes g\} \\
\text { and } \Delta: H \rightarrow H \otimes H \text { is the comultiplication of } H .
\end{gathered}
$$

If not stated otherwise, the tensor products are over the field $k$. Here $U(L)$ is the universal enveloping algebra of the Lie algebra $L$ and $k G$ is the group algebra of $G$ with coefficients in $k$. The group $G$ acts on $L$ via right conjugation: for $a \in L, g \in G$ we have $a^{g}:=g^{-1} a g$ $\in L$. The elements of $L$ are called Lie elements and the elements of $G$ group-like elements.

Let $Z=X \cup Y$ be a disjoint union of sets, $F$ the free group with basis $Y, X_{0}$ a set on which the group $F$ acts freely with $X_{0} / F \simeq X$ (that is, $X_{0}$ is the disjoint union of free $F$-orbits $\bigcup_{x \in X} x^{F}$ ) and $L_{0}$ the free Lie algebra over the field $k$ with basis $X_{\mathbf{0}}$. As defined in $[7]$

$$
H(X \mid Y)=U\left(L_{0}\right) \# k F,
$$

where the action of $F$ on $L_{0}$ via right conjugation is induced by the action of $F$ on $X_{0}$.

Let $H$ be a Hopf algebra from the category $\mathcal{X}$. We say that $H$ is finitely generated if it is finitely generated as an associative $k$-algebra. Note this is equivalent to the existence of a disjoint union $X \cup Y$ of finite sets and an epimorphism of Hopf algebras $\pi: H(X \mid Y) \rightarrow H$ that is, $H(X \mid Y)$ is a Hopf extension of the Hopf kernel $H_{1}$ of $\pi$ by $H$.

We say that $H$ is finitely presented as a Hopf algebra (or finitely presented in the category $\mathcal{X}$ ) if it is finitely generated and there is a finite set $X \cup Y$ such that for the Hopf kernel $H_{1}=h k e r(\pi)$ defined in the previous paragraph there is a finite subset $R$ of $\Omega\left(H_{1}\right)$ such that the orbits generated by the elements of $R$ via the right adjoint action of $H(X \mid Y)$ on $H_{1}$ generate $H_{1}$ as an associative $k$-algebra. Here $\Omega\left(H_{1}\right)$ denotes the kernel of the counit map $H_{1} \rightarrow k$, the right adjoint action of the group-like elements is conjugation, and the right adjoint action of the Lie elements is given by the Lie bracket. The following results can be viewed as a generalisation of the main results of [1] and will be used later on in the proof of Theorem 4 . It shows that being finitely presented in the category $\mathcal{X}$ does not depend on the choice of generators.

Theorem 2. [7, Theorem B, Cororollary 2]

(a) An element of $\mathcal{X}$ is finitely presented in the category $\mathcal{X}$ if and only if it is finitely presented as an associative $k$-algebra.

(b) Let $H$ be a Hopf algebra in the category $\mathcal{X}$ and $\pi: H(X \mid Y) \rightarrow H$ be an epimorphisms of Hopf algebras where $X \cup Y$ is a disjoint union of finite sets. Then $H$ is finitely presented in the category $\mathcal{X}$ if and only if there is 
a finite subset $R$ of the Hopf kernel $H_{1}$ of $\pi$ such that the orbits generated by the elements of $R$ via the adjoint action of $H(X \mid Y)$ generate $H_{1}$ as an associative $k$-algebra and $R$ contains only Lie elements and group-like elements - 1 .

\section{TENSOR AND EXTERIOR SQUARES}

Let $A$ be a finitely generated module over a commutative Hopf algebra $H=U\left(L_{1}\right) \# k Q$. We view $A \otimes A$ and its quotient $A \wedge A$ as right $H$-modules via the comultiplication $\Delta: H \rightarrow H \otimes H$.

THEOREM 3. The module $A \otimes A$ is finitely generated over $H$ if and only if $A \wedge A$ is finitely generated over $H$.

Proof: The above theorem is known in the case when $L_{1}=0[3$, Theorem 4.3] or $Q=1$ [6, Proposition 2.4]. In fact the proof of [6, Proposition 2.4] is for any finitely generated commutative $k$-algebra with unity $H$ that acts on $A \wedge A$ via some homomorphism of $k$-algebras $\Delta: H \rightarrow H \otimes H$ such that for some generating set (as commutative $k$-algebra with unity) $Y$ of $H$ we have $\Delta(y)=y \otimes 1+1 \otimes y$ for every $y \in Y$. A close observation of the proof of [6, Proposition 2.4] shows that in fact it uses only that for every $y \in Y$ the element $\Delta(y)$ is invariant under the action of the symmetric group on two elements that permutes the factors of $H \otimes H$. In particular the proof of [6, Propisition 2.4] holds in our case where $Y=L_{0} \cup Q_{0}, L_{0}$ is a basis of $L_{1}$ over $k, Q_{0}$ is a generating set of the Abelian group $Q$.

\section{Finite geNERATION OF TENSOR SQUARES AND FINITE PRESENTABILITY}

The purpose of this rather long section is to develop the techniques of the proof of Theorem 4. The proof of Theorem 4 will be completed in Section 4.

THEOREM 4. Let $H=U(L) \# k Q$ be a finitely generated Hopf algebra with $A$ an $Q$-invariant (via conjugation) Abelian ideal in the Lie algebra $L$ such that $R=U(L / A) \# k Q$ is commutative. If $A \otimes A$ is finitely generated over $R$ via the comultiplication $\Delta: R \rightarrow R \otimes R$, where $A$ is viewed as a right $R$-module via the adjoint action, then $H$ is finitely presented in $\mathcal{X}$.

3.1. MORE about generators and Relations for the Hopf algebra of TheOREM 4. Let $H=U(L) \# k Q$ be a Hopf algebra with $A$ an $Q$-invariant (via conjugation) Abelian ideal in the Lie algebra $L$, let $R=U(L / A) \# k Q$ be commutative, and let $H$ be finitely generated as an associative $k$-algebra. We view $A$ as a right $R$-module via the adjoint action; that is, the elements of $Q$ act by right conjugation, the elements of $L$ act via the adjoint Lie action. For $r \in R, a \in A$ we denote by $a \circ r$ the image of the action of $r$ on $a$, hence for $a \in A$

$a \circ q=q^{-1} a q$ where $q \in Q$ and $a \circ l=\left[a, l_{0}\right]$ where $l_{0} \in L, l=l_{0}+A \in L / A$. 
Under the assumptions of Theorem $4 A \otimes A$ is finitely generated over $R$ via the comultiplication $\Delta$.

LEMMA 1 . The right $R$-module $A$ is finitely generated.

Proof: Since $H$ is finitely generated there is a free resolution of the trivial right $H$-module $k$

$$
\mathcal{F}: \ldots \rightarrow F_{1} \rightarrow F_{0}=H \stackrel{\varepsilon_{H}}{\rightarrow} k \rightarrow 0
$$

with $F_{1}$ finitely generated. Here $\varepsilon_{H}$ is the counit map. We use this resolution to calculate $H_{1}(A)=\operatorname{Tor}_{1}^{U(A)}(k, k) \simeq A$ as $H_{1}\left(\mathcal{F} \otimes_{U(A)} k\right)$. Note that $H_{1}\left(\mathcal{F} \otimes_{U(A)} k\right)$ is a section of $F_{1} \otimes_{U(A)} k$ and $F_{1} \otimes_{U(A)} k$ is a finitely generated module over $H \otimes_{U(A)} k \simeq R$. As $R$ is Noetherian we deduce that $H_{1}(A) \simeq A$ is finitely generated over $R$. The fact that the action of $R$ on $A \simeq H_{1}\left(\mathcal{F} \otimes_{U(A)} k\right)$ induced by right multiplication on $\mathcal{F} \otimes_{U(A)} k$ is the right adjoint action on $A$ is proved in [7, Theorem $C]$.

Lemma 2. The Lie algebra $L / A$ is finite dimensional and for every $q \in Q, l \in L$ we have $q^{-1} l q-l \in A$.

Proof: Note that $R=U(L / A) \# k Q$ is a finitely generated commutative ring; hence $L / A$ is finite dimensional and $q^{-1} l q-l \in \operatorname{Ker}(H \rightarrow R)=H \Omega(U(A))$, where $\Omega(U(A))=A U(A)$ is the kernel of the counit map $\varepsilon_{A}: U(A) \rightarrow k$. As $q^{-1} l q-l \in L$ and $L \cap H A U(A)=A$ we deduce that $q^{-1} l q-l \in A$.

We observe that in general $Q$ is a finitely generated Abelian group, hence the direct product of a torsion-free subgroup $Q_{0}$ and a finite subgroup. If Theorem 4 is known in the case when $Q$ is torsion-free, we can deduce that in the general case, if $A \otimes A$ is a finitely generated $R$-module then $A \otimes A$ is a finitely generated $U(L) \# k Q_{0^{-}}$-module, and hence $U(L) \# k Q_{0}$ is finitely presented in $\mathcal{X}$. As $Q_{0}$ has finite index in $Q$ this implies easily that $U(L) \# k Q$ is finitely presented in $\mathcal{X}$. Then to show Theorem 4 it is sufficient to consider the case when $Q$ is torsion-free.

From now on we assume that $Q$ is a free Abelian group of rank $m$ with basis $q_{1}, \ldots, q_{m}$. Let $x_{1}, \ldots, x_{n}$ be elements of $L$ such that $\left\{x_{\mathbf{i}}^{\prime}=x_{i}+A\right\}_{1 \leqslant i \leqslant n}$ is a basis of $L / A$ as a vector space over $k$. By Lemma 1 there is a finite set $\left\{w_{1}, \ldots, w_{z}\right\}$ that generates $A$ as $R$-module, that is,

$$
A=w_{1} \circ R+\cdots+w_{z} \circ R
$$

By enlarging the set $\left\{w_{1}, \ldots, w_{z}\right\}$ if necessary we can assume that the following relations hold in $H$ :

$$
\begin{aligned}
x_{i} x_{j}-x_{j} x_{i} & =\left[x_{i}, x_{j}\right]=w_{\alpha(i, j)} \text { for } 1 \leqslant i<j \leqslant n, \\
x_{i}^{q_{j}^{f}}-x_{i} & =w_{\beta(i, j, \varepsilon)} \text { for } 1 \leqslant i \leqslant n, 1 \leqslant j \leqslant m, \varepsilon= \pm 1, \\
q_{i} q_{j} & =q_{j} q_{i} \text { for } 1 \leqslant i<j \leqslant m .
\end{aligned}
$$


3.2. Some notation. Let $X=\left\{W_{1}, \ldots, W_{z}, X_{1}, \ldots, X_{n}\right\}$ and $Y=\left\{Y_{1}, \ldots, Y_{m}\right\}$. We define $F$ as the quotient of $H(X \mid Y)$ by the two-sided ideal generated by all commutators $Y_{i} Y_{j} Y_{i}^{-1} Y_{j}^{-1}-1$ for $1 \leqslant i<j \leqslant m$. Then $H(X \mid Y)=U\left(L_{0}\right) \# k F_{0}$, where $F_{0}$ is the free group with basis $Y$ and $L_{0}$ is the free Lie algebra with basis the disjoint union of free $F_{0}$-orbits $\bigcup_{x \in X} x^{F_{0}}$ and

$$
F=U\left(L_{1}\right) \# k Q,
$$

where $Q \simeq F_{0} /\left[F_{0}, F_{0}\right]$ and $L_{1}$ is the free Lie algebra with basis the disjoint union of free $Q$-orbits $\bigcup_{x \in X} x^{Q}$. Thus $F$ is a Hopf algebra in the category $\mathcal{X}$. We identify the image of $Y_{i}$ in $Q$ with the element $q_{i}$ defined at the end of section 3.1. Now we consider a surjective homomorphism of Hopf algebras

$$
\pi: F \rightarrow H,
$$

sending $W_{i}$ to $w_{i}, X_{i}$ to $x_{i}$ and $q_{i}$ to $q_{i}$. Denote by $\widetilde{R}$ the quotient of $F$ by the associative two sided ideal generated by all $W_{i}$ 's. Note that $\widetilde{R}$ is a Hopf algebra in the category $\mathcal{X}$ and $\pi$ induces a surjective homomorphism of Hopf algebras

$$
\rho: \tilde{R} \rightarrow R \text {. }
$$

There is a $k$-linear map

$$
\eta: R \rightarrow \widetilde{R}
$$

sending $x_{1}^{z_{1}} \ldots x_{m}^{z_{m}} q_{1}^{i_{1}} \ldots q_{n}^{i_{n}}$ to $X_{1}^{z_{1}} \ldots X_{m}^{z_{m}} q_{1}^{i_{1}} \ldots q_{n}^{i_{n}}$. Note that $\eta$ is not a homomorphism of $k$-algebras. Denote by $\widetilde{\Delta}: \widetilde{R} \rightarrow \widetilde{R} \otimes \widetilde{R}$ the comultiplication of $\widetilde{R}$ and by $\Delta: R \rightarrow R \otimes R$ the comultiplication of $R$. As in [5] if not otherwise stated $f, g, \ldots$ denote elements of $R$ and $\widetilde{f}, \widetilde{g}, \ldots$ denote elements of $\widetilde{R}$. We note that by the definitions of $\widetilde{R}$ and $R$

$$
\widetilde{R} \simeq k\left[X_{1}, \ldots, X_{n}, q_{1}^{ \pm 1}, \ldots, q_{m}^{ \pm 1}\right]
$$

and

$$
R \simeq k\left[x_{1}, \ldots, x_{n}, q_{1}^{ \pm 1}, \ldots, q_{m}^{ \pm 1}\right],
$$

where both rings are associative polynomial rings where the variables $q_{1}^{ \pm 1}, \ldots, q_{m}^{ \pm 1}$ commute with each other and the variables $x_{1}, \ldots, x_{n}$ are central elements. We call

$$
w=t_{1}^{\varepsilon_{1}} \ldots t_{s}^{\varepsilon_{s}},
$$

where

$$
\varepsilon_{i}= \pm 1, t_{i} \in\left\{X_{1}, \ldots, X_{n}, q_{1}^{ \pm 1}, \ldots, q_{m}^{ \pm 1}\right\}
$$

(respectively, $t_{i} \in\left\{x_{1}, \ldots, x_{n}, q_{1}^{ \pm 1}, \ldots, q_{m}^{ \pm 1}\right\}$ ), monomial on

$$
\left\{X_{1}, \ldots, X_{n}, q_{1}^{ \pm 1}, \ldots, q_{m}^{ \pm 1}\right\}
$$


(respectively, monomial on $\left.\left\{x_{1}, \ldots, x_{n}, q_{1}^{ \pm 1}, \ldots, q_{m}^{ \pm 1}\right\}\right)$ if $w$ does not have a subword $q_{i} q_{i}^{-1}$ and $q_{i}^{-1} q_{i}$ for some $i \leqslant m$. By definition the length $|w|$ of $w$ is $s$. For $f \in R \cup \widetilde{R}$ we define $\operatorname{supp}(f)$ to be the set of all monomials in $f$ and the length $|f|$ of $f$ is

$$
|f|=\max \{|w|: w \in \operatorname{supp}(f)\} .
$$

By definition a monomial in $R \otimes R$ is $f \otimes g$ for some monomials $f, g \in R$ and has length $|f|+|g|$. A monomial in $\widetilde{R} \otimes \widetilde{R}$ is $\widetilde{f} \otimes \widetilde{g}$ for some monomials $\tilde{f}, \widetilde{g} \in \widetilde{R}$ and has length $|\widetilde{f}|+|\tilde{g}|$. If $S$ is a $k$-linear subspace of any of the following rings $R \otimes 1,1 \otimes R, \widetilde{R} \otimes 1$, $1 \otimes \widetilde{R}, R \otimes R, \widetilde{R} \otimes \widetilde{R}$ then $S_{t}$ denotes the linear subspace of all elements of $S$ of length at most $t$.

LEMMA 3. For non-negative integers $s$ and $t$ we have

$$
\Delta\left(R_{s}\right)(R \otimes R)_{t}=(R \otimes R)_{t} \Delta\left(R_{s}\right)
$$

ProOF: Note that $R \otimes R$ is a commutative ring.

3.3. SOME PROPERTIES OF THE MAP $\rho$. The following lemma is a generalisation of $[5$, Lemma 2.2].

LemMA 4 . Let $t \geqslant 2$ be a natural number. Then

1. $\operatorname{Ker}(\rho)_{t}$ is generated as a vector space by the elements of $\tilde{R}$ of the form

$$
\widetilde{p}_{1}\left(X_{j} X_{i}-X_{i} X_{j}\right) \widetilde{p}_{2} \text { and } \tilde{p}_{1}\left(X_{i} q_{k}^{\varepsilon}-q_{k}^{\varepsilon} X_{i}\right) \tilde{p}_{2}
$$

where $1 \leqslant i, j \leqslant n, 1 \leqslant k \leqslant m, \varepsilon= \pm 1, \widetilde{p}_{1}, \widetilde{p}_{2}$ are monomials in $\widetilde{R}$ and $\left|\widetilde{p}_{1}\right|+\left|\tilde{p}_{2}\right| \leqslant t-2$.

2. $\operatorname{Ker}(\rho \otimes \rho)_{t}$ is generated as a vector space by the elements of $\widetilde{R} \otimes \widetilde{R}$ of the form

$$
\begin{aligned}
& \widetilde{p}_{1}\left(\left(X_{j} X_{i}-X_{i} X_{j}\right) \otimes 1\right) \widetilde{p}_{2}, \widetilde{p}_{1}\left(1 \otimes\left(X_{j} X_{i}-X_{i} X_{j}\right)\right) \widetilde{p}_{2}, \\
& \tilde{p}_{1}\left(\left(X_{i} q_{k}^{\varepsilon}-q_{k}^{\varepsilon} X_{i}\right) \otimes 1\right) \tilde{p}_{2}, \tilde{p}_{1}\left(1 \otimes\left(X_{i} q_{k}^{\varepsilon}-q_{k}^{\varepsilon} X_{i}\right)\right) \tilde{p}_{2},
\end{aligned}
$$

where $1 \leqslant i, j \leqslant n, 1 \leqslant k \leqslant m, \varepsilon= \pm 1, \widetilde{p}_{1}, \widetilde{p}_{2}$ are monomials in $\widetilde{R} \otimes \widetilde{R}$ and $\left|\tilde{p}_{1}\right|+\left|\tilde{p}_{2}\right| \leqslant t-2$.

Proof: We give a proof of the first part of the lemma following the proof of $[\mathbf{5}$, Lemma 2.2]. We omit the proof of the second part as it is similar. Let $\tilde{f} \in \widetilde{R}_{t}$, then we can write $\tilde{f}$ in the form $\tilde{f}=\tilde{f}_{1}+\tilde{f}_{2}$ where $\tilde{f}_{1}$ is a linear combination of monomials of the form $X_{1}^{z_{1}} \ldots X_{m}^{z_{m}} q_{1}^{i_{1}} \ldots q_{n}^{i_{n}}$ and $\tilde{f}_{2}$ is a linear combination of the elements specified in part 1 of the lemma. Note that $\tilde{f}_{2} \in \operatorname{Ker}(\rho)_{t}$ and $\tilde{f}_{1}=\eta\left(f_{1}\right)$ for some $f_{1} \in R$. If $\tilde{f} \in \operatorname{Ker}(\rho)_{t}$ then $\tilde{f}_{1} \in \operatorname{Ker}(\rho)$ and $f_{1}=\rho \eta\left(f_{1}\right)=\rho\left(\tilde{f}_{1}\right)=0$, hence $\tilde{f}=\tilde{f}_{2}$.

The following lemma is an obvious corollary of the definition of $\operatorname{Ker}(\rho)_{t}$. 
LEMMA 5. Let $\tilde{p}$ be a monomial in $\widetilde{R}$ of length $t \geqslant 1$. Thus $\tilde{p}$ is a product of $t$ elements of $X \cup\left\{q_{1}^{ \pm 1}, \ldots, q_{m}^{ \pm 1}\right\}$ and let $\tilde{p}_{1}$ be a product of the same $t$ entries in $\tilde{p}$ but possibly in a different order. Then

$$
\tilde{p}-\widetilde{p}_{1} \in \operatorname{Ker}(\rho)_{t}
$$

3.4. THE ChoICE OF THE NUMBER $e_{0}$. Since $R$ is a Noetherian ring there are elements $g_{r 1}, \ldots, g_{r c}$ of $R$ such that

$$
\operatorname{Ann}_{R}\left(w_{r}\right)=g_{\mathrm{rl}} R+\cdots+g_{\mathrm{rc}} R .
$$

Since the set of generators $\left\{w_{1}, \ldots, w_{z}\right\}$ of $A$ as a right $R$-module is finite we may assume that $c$ is independent of $r$. As in [5] we have

$\mathrm{Ann}_{R \otimes R}\left(w_{r} \otimes w_{s}\right)=\left(g_{r 1} \otimes 1\right) R \otimes R+\cdots+\left(g_{r c} \otimes 1\right) R \otimes R+\left(1 \otimes g_{s 1}\right) R \otimes R+\cdots+\left(1 \otimes g_{s c}\right) R \otimes R$.

Note that the $\Delta(R)$-submodule $M_{r, s, k}$ of $A \otimes A$ generated by $\left\{\left(w_{r} \otimes w_{s}\right) \circ\left(x_{k}^{i} \otimes 1\right)\right\}_{i \geqslant 0}$, is a submodule of the finitely generated $\Delta(R)$-module $A \otimes A$. Since $\Delta(R) \simeq R$ is Noetherian there exists $l \in \mathbb{N}$ such that $M_{r, s, k}$ is generated by the elements $\left(w_{r} \otimes w_{s}\right) \circ\left(x_{k}^{i} \otimes 1\right)$ for $0 \leqslant i \leqslant l$. We may assume that $l$ is the same for all $1 \leqslant r, s \leqslant z, 1 \leqslant k \leqslant n$. Then there are elements $f_{r s k 0}, f_{r s k 1}, \ldots, f_{r s k l} \in R$ such that

$$
x_{k}^{l+1} \otimes 1+\sum_{i=0}^{l}\left(x_{k}^{i} \otimes 1\right) \Delta\left(f_{r s k i}\right) \in \operatorname{Ann}_{R \otimes R}\left(w_{r} \otimes w_{s}\right)
$$

Thus there are elements $\phi_{r s k 1}, \ldots, \phi_{r s k c}, \psi_{r s k 1}, \ldots, \psi_{r s k c} \in R \otimes R$ such that

$$
x_{k}^{l+1} \otimes 1+\sum_{i=0}^{l}\left(x_{k}^{i} \otimes 1\right) \Delta\left(f_{r s k i}\right)+\sum_{j=1}^{c}\left(g_{r j} \otimes 1\right) \phi_{r s k j}+\sum_{j=1}^{c}\left(1 \otimes g_{s j}\right) \psi_{r s k j}=0
$$

for $1 \leqslant k \leqslant n$ and $1 \leqslant r, s \leqslant z$.

Similarly considering the $\Delta(R)$-submodule of $A \otimes A$ generated by $\left(w_{r} \otimes w_{s}\right) \circ\left(q_{k}^{i} \otimes 1\right)$ for $i \geqslant 0$ we get the existence of $\hat{l} \in \mathbb{N}, \hat{f}_{r s k 0}, \hat{f}_{r s k 1}, \ldots, \hat{f}_{r s k \hat{l}} \in R, \hat{\phi}_{r s k 1}, \ldots, \hat{\phi}_{r s k c}$ and $\widehat{\psi}_{\text {rski }}, \ldots, \widehat{\psi}_{\text {rske }}$ of $R \otimes R$ such that

$$
\begin{aligned}
& q_{k}^{\hat{\imath}+1} \otimes 1+\sum_{i=0}^{\hat{\imath}}\left(q_{k}^{i} \otimes 1\right) \Delta\left(\hat{f}_{r s k i}\right) \in \operatorname{Ann}_{R \otimes R}\left(w_{r} \otimes w_{s}\right) \\
& q_{k}^{\hat{l}+1} \otimes 1+\sum_{i=0}^{\hat{l}}\left(q_{k}^{i} \otimes 1\right) \Delta\left(\hat{f}_{r s k i}\right)+\sum_{j=1}^{c}\left(g_{r j} \otimes 1\right) \widehat{\phi}_{r s k j}+\sum_{j=1}^{c}\left(1 \otimes g_{s j}\right) \hat{\psi}_{r s k j}=0
\end{aligned}
$$

for $1 \leqslant k \leqslant m$ and $1 \leqslant r, s \leqslant z$.

Similarly considering the $\Delta(R)$-submodule of $A \otimes A$ generated by $\left(w_{r} \otimes w_{s}\right) \circ\left(q_{k}^{i} \otimes 1\right)$ for $i \leqslant 0$ we get the existence of

$$
\underline{l} \in \mathbb{N}, \underline{f}_{r s k 0}, \underline{f}_{r s k(-1)}, \ldots, \underline{f}_{r s k(-1)} \in R, \underline{\phi}_{r s k 1}, \ldots, \underline{\phi}_{r s k c}
$$


and $\underline{\psi}_{r s k 1}, \ldots, \underline{\psi}_{r s k c}$ of $R \otimes R$ such that

$$
q_{k}^{-\underline{i}-1} \otimes 1+\sum_{i=-\underline{l}}^{0}\left(q_{k}^{i} \otimes 1\right) \Delta\left(\underline{f}_{r s k i}\right) \in A n n_{R \otimes R}\left(w_{r} \otimes w_{s}\right)
$$

(5) $q_{k}^{-\underline{l}-1} \otimes 1+\sum_{i=-\underline{l}}^{0}\left(q_{k}^{i} \otimes 1\right) \Delta\left(\underline{f}_{r s k i}\right)+\sum_{j=1}^{c}\left(g_{r j} \otimes 1\right) \underline{\phi}_{r s k j}+\sum_{j=1}^{c}\left(1 \otimes g_{s j}\right) \underline{\psi}_{r s k j}=0$

for $1 \leqslant k \leqslant m$ and $1 \leqslant r, s \leqslant z$.

Furthermore as $l+1, \hat{l}+1$ and $\underline{l}+1$ correspond to the cardinality of the corresponding finite generating sets of $\Delta(R)$-modules we can assume that $l=\hat{l}=\underline{l}$. Finally we define

$$
\begin{aligned}
& e_{0}=\max _{0 \leqslant i \leqslant l, 1 \leqslant r, s \leqslant z, 1 \leqslant j \leqslant c, 1 \leqslant k \leqslant m, 1 \leqslant t \leqslant n}\left\{l(n+m)+1,2\left|f_{r s t i}\right|,\right. \\
& 2\left|\hat{f}_{r s k i}\right|, 2\left|\underline{f}_{r s k(-i)}\right|,\left|\left(g_{r j} \otimes 1\right) \phi_{r s t j}\right|,\left|\left(g_{r j} \otimes 1\right) \hat{\phi}_{r s k j}\right|,\left|\left(g_{r j} \otimes 1\right) \underline{\phi}_{r s k j}\right|, \\
&\left.\left|\left(1 \otimes g_{s j}\right) \psi_{r s t j}\right|,\left|\left(1 \otimes g_{s j}\right) \hat{\psi}_{r s k j}\right|,\left|\left(1 \otimes g_{s j}\right) \underline{\psi}_{r s k j}\right|\right\} .
\end{aligned}
$$

Now we make a simple but important remark. We have defined $e_{0}$ as depending on a choice of a generating set $w_{1}, \ldots, w_{z}$ of $A$ as $R$-module. We show that if we extend this generating set to $w_{1}, \ldots, w_{z}, \ldots, w_{b}$ then we still can keep the value of $e_{0}$ the same provided the newly added generators are of the type $w_{i} \circ \lambda$ for some old generator $w_{i}$ and some $\lambda \in R$. Note that $\operatorname{Ann}_{R \otimes R}\left(w_{r} \otimes w_{s}\right) \subset \operatorname{Ann}_{R \otimes R}\left(\left(w_{r} \circ \lambda_{r}\right) \otimes\left(w_{s} \circ \lambda_{s}\right)\right)$ for $\lambda_{r}, \lambda_{s} \in R$ and by (2),(4), (5) we can use the values of $l, f, \widehat{f}, \underline{f}, \phi, \widehat{\phi}, \underline{\phi}, \psi, \widehat{\psi}, \underline{\psi}$ for the old generators $w_{i}$ 's and not introduce new ones for the new generator $w_{i} \circ \lambda$. That is, for $w_{\alpha}=w_{r} \circ \lambda_{r}$ and $w_{\beta}=w_{s} \circ \lambda_{s}$ with both $r$ and $s$ at most $z, \alpha$ or $\beta$ (or both) in $\{z+1, \ldots, b\}$, furthermore if $\alpha \leqslant z$ assume that $\alpha=r, \lambda_{r}=1$ and if $\beta \leqslant z$ assume that $\lambda_{s}=1, \beta=s$ we define

$$
\begin{array}{rlrl}
f_{\alpha \beta t i} & =f_{r s t i}, \widehat{f}_{\alpha \beta k i}=\widehat{f}_{r s k i}, \underline{f}_{\alpha \beta k i}=\underline{f}_{r s k i} & & \text { for } 1 \leqslant t \leqslant n, 1 \leqslant k \leqslant m, 1 \leqslant i \leqslant l, \\
g_{a j} & =g_{r j}, g_{\beta j}=g_{s j} & & \text { for } 1 \leqslant j \leqslant c, \\
\phi_{\alpha \beta t i} & =\phi_{r s t i}, \widehat{\phi}_{\alpha \beta k i}=\widehat{\phi}_{r s k i}, \underline{\phi}_{\alpha \beta k i}=\underline{\phi}_{r s k i} & & \text { for } 1 \leqslant i \leqslant c, 1 \leqslant t \leqslant n, 1 \leqslant k \leqslant m, \\
\psi_{\alpha \beta t i} & =\psi_{r s t i}, \widehat{\psi}_{\alpha \beta k i}=\widehat{\psi}_{r s k i}, \underline{\psi}_{\alpha \beta k i}=\underline{\psi}_{r s k i} & & \text { for } 1 \leqslant i \leqslant c, 1 \leqslant t \leqslant n, 1 \leqslant k \leqslant m, \\
\phi_{\alpha \beta t i} & =\widehat{\phi}_{\alpha \beta k i}=\underline{\phi}_{\alpha \beta k i}=\psi_{\alpha \beta t i}=\widehat{\psi}_{\alpha \beta k i}=\underline{\psi}_{\alpha \beta k i}=0 & \text { for } c+1 \leqslant i \leqslant c_{1}, 1 \leqslant t \leqslant n, 1 \leqslant k \leqslant m,
\end{array}
$$

where $c_{1}$ is the new value for the old parameter $c$. Note that by enlarging the set of generators $\left\{w_{1}, \ldots, w_{z}\right\}$ to $\left\{w_{1}, \ldots, w_{z}, \ldots, w_{b}\right\}$ the value of $c$ changes to some new natural number $c_{1} \geqslant c$. This new number $c_{1}$ can be much larger than $c$ but it is not used in the definition of $e_{0}$. We do not specify the elements $g_{\alpha j}, g_{\beta j}$ for $c+1 \leqslant j \leqslant c_{1}$ as we shall not use them. 
3.5. Fixing A finite generating SET of $A$. Let $\left\{w_{1}, \ldots, w_{z}\right\}$ be a generating set of $A$ as a right $R$-module satisfying (1) and $\pi: F \rightarrow H$ be the projection defined in subsection 3.2. We define a finite subset $E_{0}$ of the Hopf kernel $h k e r(\pi)$ as the set

$$
E_{0}=\left\{\left[X_{i}, X_{j}\right]-W_{\alpha(i, j)}, X_{r}^{q_{k}^{e}}-X_{r}-W_{\beta(r, k, \varepsilon)}\right\}_{1 \leqslant i<j \leqslant n, 1 \leqslant r \leqslant n, 1 \leqslant k \leqslant m, \varepsilon= \pm 1},
$$

where $\left[X_{i}, X_{j}\right]=X_{i} X_{j}-X_{j} X_{i}$. By reordering $\left\{w_{1}, \ldots, w_{z}\right\}$ if necessary we can assume that

$$
\{\alpha(i, j), \beta(r, k, \varepsilon)\}_{1 \leqslant i<j \leqslant n, 1 \leqslant r \leqslant n, 1 \leqslant k \leqslant m, \varepsilon= \pm 1}=\left\{1,2, \ldots, z_{0}\right\} \text { for some } z_{0} \leqslant z .
$$

For any subset $B$ of $F$ and $f_{1}, f_{2} \in F$ we write $f_{1} \equiv_{B} f_{2}$ if $f_{1}-f_{2}$ belongs to the associative two-sided ideal of $F$ generated by $B$. We use $\circ$ to denote the adjoint action in $F$, that is, $a \circ f$ is the image of $a$ under the adjoint action of $f$. By definition $\widetilde{R}$ is a quotient of $F$ and $\widetilde{R}$ can be identified with the subalgebra of $F$ generated by $\left\{X_{1}, \ldots, X_{n}, q_{1}^{ \pm 1}, \ldots, q_{m}^{ \pm 1}\right\}$.

Lemma 6. Let $\tilde{f}$ be a monomial in $\widetilde{R}$. Then there exist monomials

$$
t_{i, j, k}^{(1)}, t_{i, j, k}^{(2)}, g_{i, j, k}^{(1)}, g_{i, j, k}^{(2)}
$$

in $\widetilde{R}$ such that for $1 \leqslant i, j \leqslant n$

$$
\tilde{f}\left(X_{i} X_{j}-X_{j} X_{i}\right) \equiv_{E_{0}} \sum_{k}\left( \pm W_{\theta(i, j, k)} \circ t_{i, j, k}^{(1)}\right) g_{i, j, k}^{(1)}
$$

and for $\varepsilon= \pm 1,1 \leqslant i \leqslant n, 1 \leqslant j \leqslant m$

$$
\tilde{f}\left(X_{i} q_{j}^{\varepsilon}-q_{j}^{\varepsilon} X_{i}\right) \equiv_{E_{0}} \sum_{k}\left( \pm W_{\mu(i, j, k)} \circ t_{i, j, k}^{(2)}\right) g_{i, j, k}^{(2)}
$$

where all $\theta(i, j, k), \mu(i, j, k) \in\left\{1, \ldots, z_{0}\right\}$ and

$$
\max \left\{\left|t_{i, j, k}^{(1)}\right|,\left|g_{i, j, k}^{(1)}\right|,\left|t_{i, j, k}^{(2)}\right|-1,\left|g_{i, j, k}^{(2)}\right|-1\right\} \leqslant|\tilde{f}| .
$$

Proof: (1) We induct on the length $|\tilde{f}|$ of $\tilde{f}$, the case when $|\widetilde{f}|=0$ that is, $\tilde{f}=1$ being obvious since $X_{i} X_{j}-X_{j} X_{i} \equiv_{E_{0}} W_{a(i, j)}$ for $i<j$.

Let $|\tilde{f}| \geqslant 1$ and $\tilde{f}=\tilde{a} \tilde{f}_{0}$ where $\tilde{a} \in\left\{X_{1}, \ldots, X_{n}, q_{1}^{ \pm 1}, \ldots, q_{m}^{ \pm 1}\right\}$ and $\tilde{f}_{0} \in \widetilde{R}$ has length $|\tilde{f}|-1$. By the inductive hypothesis

$$
\tilde{f}_{0}\left(X_{i} X_{j}-X_{j} X_{i}\right) \equiv_{E_{0}} \sum_{k}\left( \pm W_{\theta(i, j, k)} \circ t_{i, j, k}^{(1)}\right) g_{i, j, k}^{(1)}
$$

where all $\theta(i, j, k) \leqslant z_{0}$ and $\max \left\{\left|t_{i, j, k}^{(1)}\right|,\left|g_{i, j, k}^{(1)}\right|\right\} \leqslant\left|\tilde{f}_{0}\right|$. Then

$$
\tilde{f}\left(X_{i} X_{j}-X_{j} X_{i}\right) \equiv_{E_{0}} \sum_{k} \tilde{a}\left( \pm W_{\theta(i, j, k)} \circ t_{i, j, k}^{(1)}\right) g_{i, j, k}^{(1)}
$$


Note that if $\tilde{a} \in\left\{q_{1}^{ \pm 1}, \ldots, q_{m}^{ \pm 1}\right\}$

$$
\begin{gathered}
\tilde{a}\left(W_{\theta(i, j, k)} \circ t_{i, j, k}^{(1)}\right) g_{i, j, k}^{(1)}=\tilde{a}\left(W_{\theta(i, j, k)} \circ t_{i, j, k}^{(1)}\right) \tilde{a}^{-1} \tilde{a} g_{i, j, k}^{(1)}=\left(W_{\theta(i, j, k)} \circ\left(t_{i, j, k}^{(1)} \tilde{a}^{-1}\right)\right) \tilde{a} g_{i, j, k}^{(1)}, \\
\max \left\{\left|t_{i, j, k}^{(1)} \tilde{a}^{-1}\right|,\left|\tilde{a} g_{i, j, k}^{(1)}\right|\right\} \leqslant\left|\tilde{f}_{0}\right|+1=|\tilde{f}| .
\end{gathered}
$$

If $\tilde{a} \in\left\{X_{1}, \ldots, X_{n}\right\}$

$$
\begin{aligned}
\tilde{a}\left(W_{\theta(i, j, k)} \circ t_{i, j, k}^{(1)}\right) g_{i, j, k}^{(1)} & =\left[\widetilde{a}, W_{\theta(i, j, k)} \circ t_{i, j, k}^{(1)}\right] g_{i, j, k}^{(1)}+\left(W_{\theta(i, j, k)} \circ t_{i, j, k}^{(1)}\right) \widetilde{a} g_{i, j, k}^{(1)} \\
& =-\left(W_{\theta(i, j, k)} \circ\left(t_{i, j, k}^{(1)} \tilde{a}\right)\right) g_{i, j, k}^{(1)}+\left(W_{\theta(i, j, k)} \circ t_{i, j, k}^{(1)}\right) \tilde{a} g_{i, j, k}^{(1)} \\
& \quad \max \left\{\left|t_{i, j, k}^{(1)} \tilde{a}\right|,\left|\tilde{a} g_{i, j, k}^{(1)}\right|,\left|t_{i, j, k}^{(1)}\right|,\left|g_{i, j, k}^{(1)}\right|\right\} \leqslant\left|\tilde{f}_{0}\right|+1=|\tilde{f}| .
\end{aligned}
$$

(2) We induct on the length $|\tilde{f}|$ of $\tilde{f}$, the case when $|\tilde{f}|=0$ that is, $\tilde{f}=1$ being obvious since

$$
X_{i} q_{j}^{\varepsilon}-q_{j}^{\varepsilon} X_{i} \equiv_{E_{0}} q_{j}^{\varepsilon} W_{\beta(i, j, \varepsilon)}=\left(W_{\beta(i, j, \varepsilon)} \circ q_{j}^{-\varepsilon}\right) q_{j}^{\varepsilon}
$$

for $i<j$.

Let $|\tilde{f}| \geqslant 1$ and $\tilde{f}=\tilde{a} \tilde{f}_{0}$ where $\tilde{a} \in\left\{X_{1}, \ldots, X_{n}, q_{1}^{ \pm 1}, \ldots, q_{m}^{ \pm 1}\right\}$ and $\tilde{f}_{0} \in \tilde{R}$ has length $|\tilde{f}|-1$. By the inductive hypothesis

$$
\tilde{f}_{0}\left(X_{i} q_{j}^{\epsilon}-q_{j}^{\varepsilon} X_{i}\right) \equiv E_{0} \sum_{k}\left( \pm W_{\theta(i, j, k)} \circ t_{i, j, k}^{(2)}\right) g_{i, j, k}^{(2)}
$$

where all $\theta(i, j, k) \leqslant z_{0}$ and $\max \left\{\left|t_{i, j, k}^{(2)}\right|,\left|g_{i, j, k}^{(2)}\right|\right\} \leqslant\left|\widetilde{f}_{0}\right|+1$. Then

$$
\tilde{f}\left(X_{i} q_{j}^{\varepsilon}-q_{j}^{\varepsilon} X_{i}\right) \equiv E_{E_{0}} \sum_{k} \tilde{a}\left( \pm W_{\theta(i, j, k)} \circ t_{i, j, k}^{(2)}\right) g_{i, j, k}^{(2)}
$$

and we can continue as in the first case.

Now we are ready to discuss the choice of a generating set of $A$ as a right $R$-module. First we start with any generating set $\left\{w_{1}, \ldots, w_{z}\right\}$ of $A$ over $R$ and use it to calculate the numbers $e_{0}, z_{0}$ and $c$ from Sections 3.4 and 3.5. Then we enlarge the generating set to

$$
\Lambda=\left\{w_{1}, \ldots, w_{z}\right\} \cup\left\{w_{i} \circ \lambda \mid 1 \leqslant i \leqslant z_{0}, \lambda \text { is a monomial in } R_{e_{0}}\right\}=\left\{w_{1}, \ldots, w_{r_{0}}\right\},
$$

for some $r_{0} \geqslant z$. Thus for $i \leqslant z_{0}$ and a monomial $\lambda \in R_{e_{0}}$ there is some natural number $\nu(i, \lambda) \leqslant r_{0}$ such that

$$
w_{i} \circ \lambda=w_{\nu(i, \lambda)}
$$

Define $F$ with respect to this generating set $\Lambda$ that is,

$F$ is the quotient of $H(X \mid Y)$ modulo the associative two-sided ideal generated by $\left\{Y_{i} Y_{j} Y_{i}^{-1} Y_{j}^{-1}-1\right\}_{1 \leqslant i<j \leqslant m}$, where $X=\left\{W_{1}, \ldots, W_{r_{0}}, X_{1}, \ldots, X_{n}\right\}, Y=\left\{Y_{1}, \ldots, Y_{m}\right\}$. 
Now we define a subset $E_{1}$ of $F$ using the subset $E_{0}$ of $F$ defined at the beginning of Section 3.5

$$
E_{1}=E_{0} \cup\left\{W_{i} \circ f-W_{\nu(i, \rho(f))}: i \leqslant z_{0}, f \text { monomial in } \widetilde{R}_{e_{0}}\right\}
$$

where $\rho: \widetilde{R} \rightarrow R$ is the homomorphism of $k$-algebras sending $X_{i}$ to $x_{i}$ and $q_{j}^{\varepsilon}$ to $q_{j}^{\varepsilon}$.

Corollary 1 . Let $\tilde{f}$ be a monomial in $\widetilde{R}$. Then there exist monomials

$$
t_{i, j, k}^{(1)} \in \widetilde{R}_{\max \left\{0,|f|-e_{0}\right\}}, t_{i, j, k}^{(2)} \in \widetilde{R}_{\max \left\{0,|f|-e_{0}+1\right\}}, g_{i, j, k}^{(1)} \in \widetilde{R}_{|\widetilde{f}|}, g_{i, j, k}^{(2)} \in \widetilde{R}_{|\widetilde{f}|+1}
$$

such that for $1 \leqslant i \neq j \leqslant n$

$$
\tilde{f}\left(X_{i} X_{j}-X_{j} X_{i}\right) \equiv_{E_{1}} \sum_{k}\left( \pm W_{\theta^{\prime}(i, j, k)} \circ t_{i, j, k}^{(1)}\right) g_{i, j, k}^{(1)}
$$

and for $\varepsilon= \pm 1,1 \leqslant i \leqslant n, 1 \leqslant j \leqslant m$

$$
\tilde{f}\left(X_{i} q_{j}^{\varepsilon}-q_{j}^{\varepsilon} X_{i}\right) \equiv_{E_{1}} \sum_{k}\left( \pm W_{\mu^{\prime}(i, j, k)} \circ t_{i, j, k}^{(2)}\right) g_{i, j, k}^{(2)}
$$

where $\mu^{\prime}(i, j, k), \theta^{\prime}(i, j, k) \in\left\{1, \ldots, r_{0}\right\}$.

Proof: 1. By Lemma 6 there exist monomials $\widetilde{t}_{i, j, k}^{(1)}, \widetilde{g}_{i, j, k}^{(1)}$ in $\widetilde{R}$ such that

$$
\begin{aligned}
\tilde{f}\left(X_{i} X_{j}-X_{j} X_{i}\right) \equiv_{E_{1}} \sum_{k}\left( \pm W_{\theta(i, j, k)} \circ \tilde{t}_{i, j, k}^{(1)}\right) \tilde{g}_{i, j, k}^{(1)}, \\
\quad \max \left\{\left|\tilde{t}_{i, j, k}^{(1)}\right|,\left|\tilde{g}_{i, j, k}^{(1)}\right|\right\} \leqslant|\tilde{f}| \text { and all } \theta(i, j, k) \leqslant z_{0} .
\end{aligned}
$$

Let $\tilde{h}_{i, j, k}^{(1)}$ be the beginning of $\tilde{t}_{i, j, k}^{(1)}$ of length $\min \left\{e_{0},\left|\tilde{t}_{i, j, k}^{(1)}\right|\right\}$ and $\widetilde{h}_{i, j, k}^{(2)}$ be the rest of $\tilde{t}_{i, j, k}^{(1)}$ that is $\widetilde{h}_{i, j, k}^{(2)}$ is obtained from $\tilde{t}_{i, j, k}^{(1)}$ after deleting the beginning $\widetilde{h}_{i, j, k}^{(1)}$. Then

$$
W_{\theta(i, j, k)} \circ \tilde{h}_{i, j, k}^{(1)} \equiv_{E_{1}} W_{\nu\left(\theta(i, j, k), \rho\left(\tilde{h}_{i, j, k}^{(1)}\right)\right)}
$$

hence

$$
\left(W_{\theta(i, j, k)} \circ \tilde{t}_{i, j, k}^{(1)}\right) \tilde{g}_{i, j, k}^{(1)} \equiv_{E_{1}}\left(W_{\nu\left(\theta(i, j, k), \rho\left(\tilde{h}_{i, j, k}^{(1)}\right)\right)} \circ \tilde{h}_{i, j, k}^{(2)}\right) \tilde{g}_{i, j, k}^{(1)}
$$

Note that

$$
\left|\tilde{h}_{i, j, k}^{(2)}\right|=\left|\tilde{t}_{i, j, k}^{(1)}\right|-\left|\widetilde{h}_{i, j, k}^{(1)}\right| \leqslant \max \left\{0,\left|\tilde{t}_{i, j, k}^{(1)}\right|-e_{0}\right\} \leqslant \max \left\{0,|\tilde{f}|-e_{0}\right\} .
$$

2. By Lemma 6 there exist monomials $\widetilde{t}_{i, j, k}^{(2)}, \widetilde{g}_{i, j, k}^{(2)}$ in $\widetilde{R}$ such that

$$
\begin{aligned}
\tilde{f}\left(X_{i} q_{j}^{\varepsilon}-q_{j}^{\varepsilon} X_{i}\right) \equiv_{E_{1}} \sum_{k}\left( \pm W_{\theta(i, j, k)} \circ \tilde{t}_{i, j, k}^{(2)}\right) \tilde{g}_{i, j, k}^{(2)} \\
\max \left\{\left|\tilde{t}_{i, j, k}^{(2)}\right|,\left|\tilde{g}_{i, j, k}^{(2)}\right|\right\} \leqslant|\tilde{f}|+1 \text { and all } \theta(i, j, k) \leqslant z_{0}
\end{aligned}
$$


Let $\tilde{h}_{i, j, k}^{(1)}$ be the beginning of $\tilde{t}_{i, j, k}^{(2)}$ of length $\min \left\{e_{0},\left|\tilde{t}_{i, j, k}^{(2)}\right|\right\}$ and $\tilde{h}_{i, j, k}^{(2)}$ be the rest of $\tilde{t}_{i, j, k}^{(2)}$ that is $\widetilde{h}_{i, j, k}^{(2)}$ is obtained from $\widetilde{t}_{i, j, k}^{(2)}$ after deleting the beginning $\widetilde{h}_{i, j, k}^{(1)}$. Then

$$
W_{\theta(i, j, k)} \circ \tilde{h}_{i, j, k}^{(1)} \equiv_{E_{1}} W_{\nu\left(\theta(i, j, k), \rho\left(\tilde{h}_{i, j, k}^{(1)}\right)\right)}
$$

hence

$$
\left(W_{\theta(i, j, k)} \circ \tilde{t}_{i, j, k}^{(2)}\right) \tilde{g}_{i, j, k}^{(2)} \Xi_{E_{1}}\left(W_{\nu\left(\theta(i, j, k), \rho\left(\tilde{h}_{i, j, k}^{(1)}\right)\right)} \circ \widetilde{h}_{i, j, k}^{(2)}\right) \tilde{g}_{i, j, k}^{(2)}
$$

Note that $\left|\widetilde{h}_{i, j, k}^{(2)}\right|=\left|\tilde{t}_{i, j, k}^{(2)}\right|-\left|\tilde{h}_{i, j, k}^{(1)}\right| \leqslant \max \left\{0,\left|\tilde{t}_{i, j, k}^{(2)}\right|-e_{0}\right\} \leqslant \max \left\{0,|\tilde{f}|-e_{0}+1\right\}$.

Finally we define

$$
\begin{aligned}
E & =E_{1} \cup\left\{W_{i} \circ \eta\left(g_{i j}\right)\right\}_{1 \leqslant i \leqslant r_{0}, 1 \leqslant j \leqslant c} \\
& \cup\left\{\left[W_{i} \circ f_{i}, W_{j} \circ f_{j}\right] \mid 1 \leqslant i, j \leqslant r_{0}, f_{i}, f_{j} \text { monomials in } \widetilde{R} \text { such that }\left|f_{i}\right|+\left|f_{j}\right| \leqslant e_{0}\right\},
\end{aligned}
$$

where $\eta: R \rightarrow \widetilde{R}$ is the $k$-linear map defined in Section 3.2. Thus

$$
\begin{aligned}
E & =\left\{\left[X_{i}, X_{j}\right]-W_{\alpha(i, j)}, X_{r}^{q_{k}^{e}}-X_{r}-W_{\beta(r, k, \varepsilon)}\right\}_{1 \leqslant i<j \leqslant n, 1 \leqslant r \leqslant n, 1 \leqslant k \leqslant m, \varepsilon= \pm 1} \\
& \cup\left\{W_{i} \circ f-W_{\nu(i, \rho(f))} \mid i \leqslant z_{0}, f \text { monomial in } \widetilde{R}_{e_{0}}\right\} \cup\left\{W_{i} \circ \eta\left(g_{i j}\right)\right\}_{1 \leqslant i \leqslant r_{0}, 1 \leqslant j \leqslant c} \\
& \cup\left\{\left[W_{i} \circ f_{i}, W_{j} \circ f_{j}\right] \mid 1 \leqslant i, j \leqslant r_{0}, f_{i}, f_{j} \text { monomials in } \widetilde{R},\left|f_{i}\right|+\left|f_{j}\right| \leqslant e_{0}\right\} .
\end{aligned}
$$

Note that in the definition of $E$ we use the old value of $c$ obtained before enlarging the generating set of $A$.

3.6. Some COMMUtator calculations. Our main theorem easily follows from the following Theorem 5 . The proof is split in several small lemmas. We remind the reader that we use $\circ$ to denote the adjoint action in $F$, that is, $a \circ f$ is the image of $a$ under the adjoint action of $f$.

THEOREM 5. For any $1 \leqslant i, j \leqslant r_{0}$ and $f_{i}, f_{j}$ monomials in $\widetilde{R}$ we have

$$
\left[W_{i} \circ f_{i}, W_{j} \circ f_{j}\right] \equiv_{E} 0
$$

An induction on $e \geqslant e_{0}$ will be used to show that

$$
\left[W_{i} \circ f_{i}, W_{j} \circ f_{j}\right] \equiv_{E} 0 \text { for monomials with }\left|f_{i}\right|+\left|f_{j}\right| \leqslant e
$$

We assume (7) holds for some fixed value of $e$ and aim to show that

$$
\left[W_{i} \circ f_{i}, W_{j} \circ f_{j}\right] \equiv_{E} 0 \text { for }\left|f_{i}\right|+\left|f_{j}\right|=e+1
$$

LEMMA 7. $W_{i} \circ \tilde{f} \equiv_{E} 0$ for all $i \leqslant r_{0}, \tilde{f} \in \operatorname{Ker}(\rho)_{e+2}$. 
ProOF: By Lemma 4(1) $\operatorname{Ker}(\rho)_{e+2}$ is generated as a vector space by the elements of $\widetilde{R}$ of the form

$$
\tilde{p}_{1}\left(X_{j} X_{r}-X_{r} X_{j}\right) \tilde{p}_{2} \text { and } \tilde{p}_{1}\left(X_{j} q_{k}^{\varepsilon}-q_{k}^{\varepsilon} X_{j}\right) \tilde{p}_{2} \text {, }
$$

where $1 \leqslant j<r \leqslant n, 1 \leqslant k \leqslant m, \varepsilon= \pm 1, \tilde{p}_{1}, \widetilde{p}_{2}$ are monomials in $\widetilde{R}$ and $\left|\tilde{p}_{1}\right|+\left|\widetilde{p}_{2}\right| \leqslant e$. Then we can assume that either $\tilde{f}=\tilde{p}_{1}\left(X_{j} X_{r}-X_{r} X_{j}\right) \tilde{p}_{2}$ or

$$
\tilde{f}=\tilde{p}_{1}\left(X_{j} q_{k}^{\varepsilon}-q_{k}^{\varepsilon} X_{j}\right) \tilde{p}_{2} .
$$

If $\tilde{f}=\tilde{p}_{1}\left(X_{j} X_{r}-X_{r} X_{j}\right) \tilde{p}_{2}$ using that $\left|\tilde{p}_{1}\right| \leqslant e$

$$
W_{i} \circ \tilde{f}=W_{i} \circ\left(\tilde{p}_{1}\left(X_{j} X_{r}-X_{r} X_{j}\right) \tilde{p}_{2}\right) \equiv_{E}\left[W_{i} \circ \tilde{p}_{1}, W_{\alpha(j, r)}\right] \circ \tilde{p}_{2} \equiv_{E} 0 \circ \tilde{p}_{2}=0 .
$$

If $\tilde{f}=\tilde{p}_{1}\left(X_{j} q_{k}^{\varepsilon}-q_{k}^{\varepsilon} X_{j}\right) \tilde{p}_{2}$

$$
\begin{aligned}
W_{i} \circ \tilde{f}=W_{i} \circ\left(\widetilde{p}_{1}\left(X_{j} q_{k}^{\varepsilon}-q_{k}^{\varepsilon} X_{j}\right) \widetilde{p}_{2}\right)= & W_{i} \circ\left(\widetilde{p}_{1}\left(X_{j}-X_{j}^{q_{k}^{-\varepsilon}}\right) q_{k}^{\varepsilon} \widetilde{p}_{2}\right) \equiv_{E} \\
& {\left[W_{i} \circ \widetilde{p}_{1},-W_{\beta(j, k,-\varepsilon)} \circ \circ\left(q_{k}^{\varepsilon} \tilde{p}_{2}\right) \equiv_{E} 0 \circ\left(q_{k}^{\varepsilon} \widetilde{p}_{2}\right)=0 .\right.}
\end{aligned}
$$

Let $W$ be the associative two-sided ideal of $F$ generated by $W_{1}, \ldots, W_{r_{0}}$ and

$$
\chi: W \otimes W \rightarrow[W, W]
$$

be the homomorphism of right $\widetilde{\Delta}(\widetilde{R})$-modules sending $m_{1} \otimes m_{2}$ to $\left[m_{1}, m_{2}\right]$. We view $W$ as a right $\widetilde{R}$-module via the adjoint action, $[W, W]$ is a $\widetilde{R}$-submodule of $W$ and $W \otimes W$ is a right $\widetilde{\Delta}(\widetilde{R})$-module via the comultiplication $\widetilde{R} \rightarrow \widetilde{R} \otimes \widetilde{R}$. Abusing the notations we write $\circ$ for both the right adjoint action in $F$ and in $F \otimes F$.

LEMMA 8. Let $\tilde{\zeta} \in \operatorname{Ker}(\rho \otimes \rho)_{e+e_{0}}$. Then for $1 \leqslant r, s \leqslant r_{0}$

$$
\chi\left(\left(W_{r} \otimes W_{s}\right) \circ \tilde{\zeta}\right) \equiv_{E} 0 .
$$

PROOF: By Lemma $4(2) \operatorname{Ker}(\rho \otimes \rho)_{e+e_{0}}$ is spanned by

$$
\tilde{\mu}_{1}\left(\left(X_{j} X_{i}-X_{i} X_{j}\right) \otimes 1\right) \tilde{\mu}_{2}, \tilde{\mu}_{1}\left(1 \otimes\left(X_{j} X_{i}-X_{i} X_{j}\right)\right) \tilde{\mu}_{2}, \tilde{\mu}_{1}\left(\left(X_{i} q_{j}^{\varepsilon}-q_{j}^{\varepsilon} X_{i}\right) \otimes 1\right) \tilde{\mu}_{2}
$$

and

$$
\tilde{\mu}_{1}\left(1 \otimes\left(X_{i} q_{j}^{\varepsilon}-q_{j}^{\varepsilon} X_{i}\right)\right) \tilde{\mu}_{2}
$$

where $\tilde{\mu}_{1}, \tilde{\mu}_{2}$ are monomials in $\widetilde{R} \otimes \widetilde{R}$ with $\left|\widetilde{\mu}_{1}\right|+\left|\tilde{\mu}_{2}\right| \leqslant e+e_{0}-2$ and $\varepsilon= \pm 1$. As the problem is symmetric with respect to swapping the components in $W \otimes W$ it is sufficient to consider only the cases $\tilde{\zeta}=\tilde{\mu}_{1}\left(\left(X_{j} X_{i}-X_{i} X_{j}\right) \otimes 1\right) \tilde{\mu}_{2}$ and $\tilde{\zeta}=\tilde{\mu}_{1}\left(\left(X_{i} q_{j}^{\varepsilon}-q_{j}^{\varepsilon} X_{i}\right) \otimes 1\right) \tilde{\mu}_{2}$. We write $\tilde{\mu}_{1}=\tilde{p}_{1} \otimes \tilde{q}_{1}, \tilde{\mu}_{2}=\tilde{p}_{2} \otimes \tilde{q}_{2}$, where $\tilde{p}_{1}, \tilde{p}_{2}, \widetilde{q}_{1}, \tilde{q}_{2}$ are monomials in $\widetilde{R}$.

First we consider the case $\tilde{\zeta}=\tilde{\mu}_{1}\left(\left(X_{j} X_{i}-X_{i} X_{j}\right) \otimes 1\right) \tilde{\mu}_{2}$. Note that by Corollary 1

$$
\tilde{p}_{1}\left(X_{i} X_{j}-X_{j} X_{i}\right) \equiv_{E} \sum_{k}\left( \pm W_{\theta^{\prime}(i, j, k)} \circ \tilde{t}_{k}\right) \tilde{p}_{3, k}
$$


where $\widetilde{t}_{k}, \widetilde{p}_{3, k}$ are monomials in $\widetilde{R}$ with $\left|\widetilde{t}_{k}\right| \leqslant \max \left\{\left|\widetilde{p}_{1}\right|-e_{0}, 0\right\} \leqslant \max \{e-2,0\} \leqslant e$ and $\left|\tilde{p}_{3, k}\right| \leqslant\left|\widetilde{p}_{1}\right|$. Then

$$
\begin{aligned}
\chi\left(\left(W_{r} \otimes W_{s}\right) \circ \widetilde{\mu}_{1}\right. & \left.\left(\left(X_{i} X_{j}-X_{j} X_{i}\right) \otimes 1\right) \tilde{\mu}_{2}\right) \\
& =\left[W_{r} \circ\left(\widetilde{p}_{1}\left(X_{i} X_{j}-X_{j} X_{i}\right) \widetilde{p}_{2}\right), W_{s} \circ\left(\widetilde{q}_{1} \widetilde{q}_{2}\right)\right] \\
& \equiv_{E}\left[W_{r} \circ\left(\sum_{k}\left( \pm W_{\theta^{\prime}(i, j, k)} \circ \widetilde{t}_{k}\right) \tilde{p}_{3, k} \tilde{p}_{2}\right), W_{s} \circ\left(\widetilde{q}_{1} \tilde{q}_{2}\right)\right] \\
& =\left[\sum_{k}\left[W_{r} \pm W_{\theta^{\prime}(i, j, k)} \circ \tilde{t}_{k}\right] \circ\left(\widetilde{p}_{3, k} \widetilde{p}_{2}\right), W_{s} \circ\left(\widetilde{q}_{1} \widetilde{q}_{2}\right)\right] \equiv_{E} 0 .
\end{aligned}
$$

The latest equivalence holds since $\left|\widetilde{t}_{k}\right| \leqslant e$ and then $\left[W_{r}, \pm W_{\theta^{\prime}(i, j, k)} \circ \widetilde{t}_{k}\right] \equiv_{E} 0$.

Now we consider the case $\widetilde{\zeta}=\widetilde{\mu}_{1}\left(\left(X_{i} q_{j}^{\varepsilon}-q_{j}^{\varepsilon} X_{i}\right) \otimes 1\right) \widetilde{\mu}_{2}$. Using again Corollary 1

$$
\tilde{p}_{1}\left(X_{i} q_{j}^{\varepsilon}-q_{j}^{\varepsilon} X_{i}\right) \equiv_{E} \sum_{k}\left( \pm W_{\mu^{\prime}(i, j, k)} \circ \tilde{t}_{k}\right) \widetilde{p}_{3, k}
$$

where $\tilde{t}_{k}, \tilde{p}_{3, k}$ are monomials in $\widetilde{R}$ with $\left|\widetilde{t}_{k}\right| \leqslant \max \left\{\left|\tilde{p}_{1}\right|-e_{0}+1,0\right\} \leqslant \max \{e-1,0\} \leqslant e$ and $\left|\tilde{p}_{3, k}\right| \leqslant\left|\tilde{p}_{1}\right|+1$. Then

$$
\begin{aligned}
\chi\left(\left(W_{r} \otimes W_{s}\right) \circ \widetilde{\mu}_{1}\right. & \left.\left(\left(X_{i} q_{j}^{\varepsilon}-q_{j}^{\varepsilon} X_{i}\right) \otimes 1\right) \tilde{\mu}_{2}\right) \\
& =\left[W_{r} \circ\left(\tilde{p}_{1}\left(X_{i} q_{j}^{\varepsilon}-q_{j}^{\varepsilon} X_{i}\right) \tilde{p}_{2}\right), W_{s} \circ\left(\widetilde{q}_{1} \tilde{q}_{2}\right)\right] \\
& \equiv_{E}\left[W_{r} \circ\left(\sum_{k}\left( \pm W_{\mu^{\prime}(i, j, k)} \circ \widetilde{t}_{k}\right) \widetilde{p}_{3, k} \tilde{p}_{2}\right), W_{s} \circ\left(\widetilde{q}_{1} \widetilde{q}_{2}\right)\right] \\
& =\left[\sum_{k}\left[W_{r}, \pm\left(W_{\mu^{\prime}(i, j, k)} \circ \widetilde{t}_{k}\right)\right] \circ\left(\tilde{p}_{3, k} \tilde{p}_{2}\right), W_{s} \circ\left(\widetilde{q}_{1} \tilde{q}_{2}\right)\right] \\
& \equiv_{E}\left[\sum_{k} 0 \circ\left(\tilde{p}_{3, k} \tilde{p}_{2}\right), W_{s} \circ\left(\tilde{q}_{1} \tilde{q}_{2}\right)\right]=0 .
\end{aligned}
$$

LEMMA 9. If for every monomial $\tilde{f}$ in $\widetilde{R}$ of length $e+1$ we have $\left[W_{i} \circ \tilde{f}, W_{j}\right] \equiv_{E} 0$ then for all monomials $\tilde{f}_{i}, \tilde{f}_{j}$ in $\widetilde{R}$ with $\left|\tilde{f}_{i}\right|+\left|\tilde{f}_{j}\right|=e+1$ we have $\left[W_{i} \circ \tilde{f}_{i}, W_{j} \circ \widetilde{f}_{j}\right] \equiv_{E} 0$.

Proof: We use induction on $\left|\tilde{f}_{j}\right|$. If $\left|\tilde{f}_{j}\right| \geqslant 1$ then $\tilde{f}_{j}=\tilde{g} X_{t}$ or $\tilde{f}_{j}=\tilde{g} q_{t}^{\varepsilon}$ for some $\varepsilon= \pm 1,|\tilde{g}|=\left|\tilde{f}_{j}\right|-1$. In the first case as $X_{t}$ is a Lie element

$$
\begin{aligned}
& {\left[W_{i} \circ \tilde{f}_{i}, W_{j} \circ \tilde{f}_{j}\right]=\left[W_{i} \circ \tilde{f}_{i}, W_{j} \circ\left(\tilde{g} X_{t}\right)\right]} \\
& \quad=\left[W_{i} \circ \tilde{f}_{i}, W_{j} \circ \tilde{g}\right] \circ X_{t}-\left[W_{i} \circ\left(\tilde{f}_{i} X_{t}\right), W_{j} \circ \tilde{g}\right] \equiv_{E}-\left[W_{i} \circ\left(\tilde{f}_{i} X_{t}\right), W_{j} \circ \tilde{g}\right],
\end{aligned}
$$

where the last equivalence follows from the fact that $\left|\tilde{f}_{i}\right|+|\tilde{g}|=e$, hence

$$
\left[W_{i} \circ \tilde{f}_{i}, W_{j} \circ \tilde{g}\right] \equiv_{E} 0 .
$$


In the second case

$$
\left[W_{i} \circ \tilde{f}_{i}, W_{j} \circ \tilde{f}_{j}\right]=\left[W_{i} \circ \tilde{f}_{i}, W_{j} \circ\left(\tilde{g} q_{t}^{\varepsilon}\right)\right]=\left[W_{i} \circ\left(\tilde{f}_{i} q_{t}^{-\epsilon}\right), W_{j} \circ \tilde{g}\right] \circ q_{t}^{\varepsilon},
$$

hence $\left[W_{i} \circ \tilde{f}_{i}, W_{j} \circ \tilde{f}_{j}\right] \equiv_{E} 0$ if and only if $\left[W_{i} \circ \tilde{f}_{i} q_{t}^{-\varepsilon}, W_{j} \circ \tilde{g}\right] \equiv_{E} 0$. This completes the inductive step.

THEOREM 6. Let $f$ be a monomial in $\widetilde{R}$ such that $|\tilde{f}|=e+1$. Then for $1 \leqslant r, s \leqslant r_{0}$

$$
\left[W_{r} \circ \tilde{f}, W_{s}\right] \equiv_{E} 0 .
$$

Proof: By Lemma 5 if $\tilde{f}_{1}$ is obtained from $\tilde{f}$ by reordering of the entries then $\tilde{f}-\tilde{f}_{1}=\tilde{\lambda}_{1} \in \operatorname{Ker}(\rho)_{e+1}$. By Lemma $7\left[W_{r} \circ \tilde{\lambda}_{1}, W_{s}\right] \equiv_{E} 0$. Hence

$$
\left[W_{r} \circ \tilde{f}, W_{s}\right] \equiv_{E} 0 \text { if and only if }\left[W_{r} \circ \widetilde{f}_{1}, W_{s}\right] \equiv_{E} 0 \text {. }
$$

Let $z_{i}$ be the number of entries of $X_{i}$ in $f$ and $m_{i}$ be the sum of all possible $\varepsilon= \pm 1$ such that $q_{i}^{\varepsilon}$ is a subword of $\tilde{f}$. Since $e_{0} \geqslant l(n+m)+1$ either there is some

$$
z_{i} \geqslant l+1
$$

or there is some

$$
\left|m_{i}\right| \geqslant l+1
$$

or there is a reordering $\tilde{f}_{1}$ of $\tilde{f}$ such that a cancelation $q_{i}^{\varepsilon} q_{i}^{-\varepsilon}$ occurs in $\tilde{f}_{1}$, hence

$$
\left|\tilde{f}_{1}\right|<|\tilde{f}| \text {. }
$$

In the last case we have $\left|\tilde{f}_{1}\right| \leqslant e$, hence $\left[W_{r} \circ \tilde{f}_{1}, W_{s}\right] \equiv_{E} 0$ and we are done. Then we can assume that we are in one of the first two cases and consider some reordering $\tilde{f}_{1}$ of $\tilde{f}$ such that

$$
\tilde{f}_{1}=\widetilde{b}^{l+1} \tilde{h}
$$

for some monomial $\tilde{h} \in \operatorname{Im}(\eta) \subset \widetilde{R}$ that does not start with $\tilde{b}^{-1}$, where

$$
\widetilde{b} \in\left\{X_{1}, \ldots, X_{n}, q_{1}^{ \pm 1}, \ldots, q_{m}^{ \pm 1}\right\} .
$$

We write $h$ for $\rho(\widetilde{h})$ and $b$ for $\rho(\tilde{b})$. Note that $\widetilde{h} \in \widetilde{R}_{e-l}$, hence $h \in R_{e-l}$. Following [5] we multiply (3) or (4) or (5) depending on the value of $b$ with $h \otimes 1$ and obtain for the fixed $r, s \in\left\{1, \ldots, r_{0}\right\}$

$$
\left(b^{b+1} h\right) \otimes 1+\alpha+\sum_{j=1}^{c}\left(g_{r j} \otimes 1\right) \beta_{r s k j}+\sum_{j=1}^{c}\left(1 \otimes g_{s j}\right) \gamma_{r s k j}=0
$$


where

$$
\begin{aligned}
& b=x_{k}, \alpha=\sum_{i=0}^{l}\left(b^{i} \otimes 1\right) \Delta\left(f_{r s k i}\right)(h \otimes 1), \beta_{r s k j}=\phi_{r s k j}(h \otimes 1), \gamma_{r s k j}=\psi_{r s k j}(h \otimes 1) \quad \text { or } \\
& b=q_{k}, \alpha=\sum_{i=0}^{l}\left(b^{i} \otimes 1\right) \Delta\left(\widehat{f}_{r s k i}\right)(h \otimes 1), \beta_{r s k j}=\widehat{\phi}_{r s k j}(h \otimes 1), \gamma_{r s k j}=\widehat{\psi}_{r s k j}(h \otimes 1) \quad \text { or } \\
& b=q_{k}^{-1}, \alpha=\sum_{i=-l}^{0}\left(b^{i} \otimes 1\right) \Delta\left(\underline{f}_{r s k i}\right)(h \otimes 1), \beta_{r s k j}=\underline{\phi}_{r s k j}(h \otimes 1), \gamma_{r s k j}=\underline{\psi}_{r s k j}(h \otimes 1)
\end{aligned}
$$

Note that the choice of $e_{0}$ together with Lemma 3 imply

(9) $\alpha \in(R \otimes R)_{l} \Delta\left(R_{\mathrm{e}_{0} / 2}\right)(R \otimes R)_{e-1}=(R \otimes R)_{e} \Delta\left(R_{e_{0} / 2}\right) \subseteq(R \otimes R)_{e+e_{0}}$,

$\left(g_{r j} \otimes 1\right) \beta_{r s k j},\left(1 \otimes g_{s j}\right) \gamma_{r s k j} \in(R \otimes R)_{e_{0}+e-l} \subseteq(R \otimes R)_{e+e_{0}}$.

We lift (8) in $\widetilde{R} \otimes \widetilde{R}$ to find $\widetilde{\tau} \in \widetilde{R} \otimes \widetilde{R}$ such that

$$
\tilde{\tau}=\tilde{f}_{1} \otimes 1+\tilde{\alpha}+\sum_{j}\left(\eta\left(g_{r j}\right) \otimes 1\right) \tilde{\beta}_{r s k j}+\sum_{j}\left(1 \otimes \eta\left(g_{s j}\right)\right) \tilde{\gamma}_{r s k j},
$$

where $\tilde{\beta}_{r s k j}=(\eta \otimes \eta)\left(\beta_{r s k j}\right), \tilde{\gamma}_{r s k j}=(\eta \otimes \eta)\left(\gamma_{r s k j}\right)$ and by (9) we can choose

$$
\tilde{\alpha} \in(\widetilde{R} \otimes \widetilde{R})_{e} \widetilde{\Delta}\left(\widetilde{R}_{e_{0} / 2}\right) \subseteq(\widetilde{R} \otimes \widetilde{R})_{e+e_{0}},
$$

such that $(\rho \otimes \rho)(\widetilde{\alpha})=\alpha$. Note that

$$
\left(\eta\left(g_{r j}\right) \otimes 1\right) \tilde{\beta}_{r s k j},\left(1 \otimes \eta\left(g_{s j}\right)\right) \tilde{\gamma}_{r s k j} \in(\widetilde{R} \otimes \widetilde{R})_{e+e_{0}} .
$$

Then

$$
\tilde{\tau} \in \operatorname{Ker}(\rho \otimes \rho)_{e+e_{0}}
$$

Note that

$$
\left[W_{\tau} \circ \tilde{f}_{1}, W_{s}\right] \equiv_{E} 0
$$

is equivalent to

$$
\chi\left(\left(W_{r} \otimes W_{s}\right) \circ\left(\tilde{f}_{1} \otimes 1\right)\right) \equiv_{E} 0 .
$$

Then by (10) it is sufficient to show that

$$
\chi\left(\left(W_{r} \otimes W_{s}\right) \circ \tilde{\zeta}\right) \equiv_{E} 0 \text { for } \tilde{\zeta} \in\left\{\tilde{\tau}, \tilde{\alpha},\left(\eta\left(g_{r j}\right) \otimes 1\right) \tilde{\beta}_{r s k j},\left(1 \otimes \eta\left(g_{s j}\right)\right) \widetilde{\gamma}_{r s k j}\right\} .
$$

These are covered by the following 3 cases:

1. $\tilde{\zeta} \in \operatorname{Ker}(\rho \otimes \rho)_{e+e_{0}}$ for $\tilde{\zeta}=\tilde{\tau}$;

2. $\tilde{\zeta} \in(\widetilde{R} \otimes \widetilde{R})_{e} \tilde{\Delta}(\tilde{R})$ for $\tilde{\zeta}=\tilde{\alpha}$; 
3. $\tilde{\zeta} \in\left(\eta\left(g_{r j}\right) \otimes 1\right) \widetilde{R} \otimes \widetilde{R}$ or $\left(1 \otimes \eta\left(g_{s j}\right)\right)(\widetilde{R} \otimes \widetilde{R})$ for $\tilde{\zeta} \in\left\{\left(\eta\left(g_{r j}\right) \otimes 1\right) \tilde{\beta}_{r s k j}\right.$, $\left(1 \otimes \eta\left(g_{s j}\right) \widetilde{\gamma}_{r s k j}\right\}$.

Case 1 follows from Lemma 8. Case 2 follows by (7) and the fact that $\chi$ is a homomorphism of right $\widetilde{\Delta}(\widetilde{R})$-modules. Case 3 follows from the fact that $W_{r} \circ \eta\left(g_{r j}\right) \in E$, hence $W_{r} \circ \eta\left(g_{r j}\right) \equiv_{E} 0$. Similarly $W_{s} \circ \eta\left(g_{s j}\right) \equiv_{E} 0$. This completes the proof of Theorem 6.

Note that Lemma 9 and Theorem 6 complete the proof of Theorem 5 .

\section{Proofs of Theorem 4 AND TheOREM 1}

4.1. Proof of Theorem 4. We remind the reader that by Theorem 5 there is a finite subset $E$ of $F=U\left(L_{1}\right) \# k Q$ such that

$$
\left[W_{i} \circ f_{i}, W_{j} \circ f_{j}\right] \equiv_{E} 0
$$

for all $i, j \leqslant r_{0}$ and all monomials $f_{i}, f_{j}$ in $\widetilde{R}$. Let $H_{1}$ be the associative ring quotient of $F$ modulo the two-sided ideal of $F$ generated by $E$. Note that the elements of $E$ are in fact Lie elements of $F$, that is, for the comultiplication $\Delta_{F}$ of $F$ we have $\Delta_{F}(r)=r \otimes 1+1 \otimes r$ for $r \in E$. Then $H_{1}$ is a Hopf algebra in the category $\mathcal{X}$ that is, $H_{1} \simeq U\left(L_{0}\right) \# k Q$, where $L_{0}$ is a Lie algebra quotient of $L_{1}$.

Let $B_{0}$ be the Lie subalgebra of $L_{0}$ such that $B_{0}$ is generated as a Lie algebra by the $H_{1}$-orbits of all images of $\left\{W_{i}\right\}_{1 \leqslant i \leqslant r_{0}}$ in $H_{1}$, where $H_{1}$ acts via the right adjoint action. Then by Theorem $5 B_{0}$ is an Abelian Lie algebra. Furthermore note that $H_{1}$ is a Hopf extension of the universal enveloping algebra $U\left(B_{0}\right)$ by $R$, that is, there is a short exact sequence of Hopf algebras $U\left(B_{0}\right) \rightarrow H_{1} \rightarrow R$ sending the image of $X_{i}$ in $H_{1}$ to $x_{i} \in R$ and the image of $q_{j}^{\varepsilon}$ in $H_{1}$ to $q_{j}^{\varepsilon} \in R$ for $\varepsilon= \pm 1$. Since $H_{1}$ acts on $B_{0}$ via the right adjoint action and $B_{0}$ is Abelian, $B_{0}$ acts trivially. This induces an action of $R$ on $B_{0}$. Then there is a homomorphism of right $R$-modules

$$
\pi: B_{0} \rightarrow A
$$

sending the image of $W_{i}$ in $B_{0}$ to $w_{i} \in A$, where $R$ acts on $A$ via the right adjoint action induced from the short exact sequence of Hopf algebras $U(A) \rightarrow H \rightarrow R$. Since $R$ is Noetherian Ker $\pi$ is finitely generated as an $R$-module. Then there exists a finite subset $\widetilde{E}$ of $L_{1}$ such that the associative ring that is the quotient of $F$ modulo the associative two-sided ideal generated by $E \cup \widetilde{E}$ is isomorphic to $H$. Then by the definition of $F$ and Theorem 2(b) $H$ is finitely presented in the category $\mathcal{X}$.

4.2. Proof of Theorem 1. By [7, Proposition 2] every finitely presented Hopf algebra in the category $\mathcal{X}$ is of homological type $F P_{2}$, thus 1. implies 2. By [7, Corollary 3] if $H$ is a Hopf algebra in $\mathcal{X}$ of type $F P_{m}$ such that $H$ is a Hopf extension of $H_{1}=U\left(L_{1}\right)$ by a Hopf algebra $H_{2}$ in $\mathcal{X}, L_{1}$ is an Abelian Lie algebra and $H_{2}$ is right Noetherian as 
an associative $k$-algebra then the $m$ th homology $H_{m}\left(L_{1}\right) \simeq \wedge^{m} L_{1}$ of the Lie algebra $L_{1}$ is finitely generated as a right $\mathrm{H}_{2}$-module via the iterated comultiplication $\mathrm{H}_{2} \rightarrow \otimes^{m} \mathrm{H}_{2}$, where we view $L_{1}$ as a right $H_{2}$-module via the adjoint action. Applying this result for $m=2, L_{1}=A$ and $H_{2}=R$ we get that 2 . implies 3 . Finally 3 . implies 4 . follows from Theorem 3 and 4 . implies 1 follows from Theorem 4.

\section{REFERENCES}

[1] G. Baumslag, 'Some remarks on finitely presented algebras', J. Pure Appl. Algebra 8 (1976), 187-196.

[2] M. Bestvina and N. Brady, 'Morse theory and finiteness properties of groups', Invent. Math. 129 (1997), 445-470.

[3] R. Bieri and J.R.J. Groves, 'Metabelian groups of type $F P_{\infty}$ are virtually of type $F P$ ', Proc. London Math. Soc. (3) 45 (1982), 365-384.

[4] R. Bieri and R. Strebel, 'Valuations and finitely presented metabelian groups', Proc. London Math. Soc. (3) 41 (1980), 439-464.

[5] R. Bryant and J.R.J. Groves, 'Finite presentation of abelian-by-finite dimensional Lie algebras', J. London Math. Soc. (2) 60 (1999), 45-57.

[6] R. Bryant and J.R.J. Groves, 'Finitely presented Lie algebras', J. Algebra 218 (1999), $1-25$.

[7] D.H. Kochloukova, 'Finite presentability and the homological type $F P_{m}$ for a class of Hopf algebras', Comm. Algebra (to appear).

[8] S. Montgomery, Hopf algebras and their actions on rings, CBMS Regional Conference Series in Mathematics 82 (American Mathematical Society, Providence, R.I., 1993).

IMECC-UNICAMP

Cx. P. 6065

13083-970 Campinas, SP

Brazil 